\title{
Ultrasound Assessment of Fetal Adrenal Gland in Term and Preterm Labor Cases
}

\author{
Heba Maged Abo Shady ${ }^{1}$, Mohamed Ahmed Samy Kandil ${ }^{1}$, \\ Ashraf Anas Zytoon ${ }^{2}$, Shaimaa Mahmoud Youssef ${ }^{1}$ \\ Departments of ${ }^{1}$ Gynecology \& Obstetrics and ${ }^{2}$ Radiodiagnosis, Faculty of Medicine - Menoufia University, Egypt \\ *Corresponding author: Shaimaa Mahmoud Youssef, Mobile: (+20)01063603679, \\ E-Mail: shaimaayoussef606@gmail.com
}

\begin{abstract}
Background: Preterm birth (PTB) remains a major cause of perinatal morbidity and mortality worldwide. Compared with term deliveries, early PTB $(<34$ weeks' gestation) carries a 7 -fold increased risk of neonatal death.

Objective: To compare the Fetal Zone Depth (FZD) of fetal adrenal gland in term and preterm labor cases

Patients and Methods: Thirty-three preterm pregnant women at 29-36 weeks of gestation with single pregnancy and clinical diagnosis of preterm labor and control group of 33 pregnant women at 37-40 weeks with term pregnancy were included in this study. FZD and Total Gland Depth (TGD) of fetal adrenal gland were ultrasonographically measured and FZD/TGD ratios were calculated and compared between the two groups.

Results: No difference was found between the two groups in respect of age and number of pregnancies $(\mathrm{P}>0.05)$. Yet, preterm birth history rupture of membrane, cervical dilatation and mode of delivery were higher in preterm group ( $\mathrm{P}$ $<0.05)$. Fetal adrenal gland FZD/TGD ratio was statistically significantly higher in preterm group compared to the term group $(52 \% \pm 5 \%$ vs. $29 \% \pm 9 \%$; $\mathrm{P}<0.001)$.

Conclusion: The growth in FZ as a fetal adaptation mechanism in increased fetal stress in preterm labor cases was at a significant level. Once supported by more comprehensive studies, we think that this result would be beneficial in the prediction of preterm labor in clinical practice.
\end{abstract}

Keywords: Preterm labor, Adrenal gland, Fetal zone, Ultrasonography.

\section{INTRODUCTION}

Identification of women at risk for PTB is one of the critical prerequisites for effective intervention and improvement in outcome. For the last several decades, significant effort has been focused toward discovery of an accurate method to predict PTB.

Traditional predictors such as obstetric risk factors and clinical presentation are helpful but seldom completely define the population that will truly deliver preterm ${ }^{(\mathbf{1})}$. Approximately $20-30 \%$ of the preterm births (i.e. $1-4 \%$ of all births) are caused by medical and obstetric reasons. While, $70-80 \%$ of these births may be linked to spontaneous idiopathic reasons associated or not associated with premature rupture of membranes (PROM) ${ }^{(2)}$. Preeclampsia, fetal distress, Fetal Growth Retardation (FGR), abruption placenta and fetal death may be referred to as the medical and obstetric causes ${ }^{(\mathbf{3})}$.

The prediction and prevention of preterm births are among the major issues of obstetrics. Prediction and prevention of preterm births by determining the risk factors would be the most appropriate approach with a view to reduce the preterm birth rates. Risk scoring systems, digital examination of the cervix, biochemical markers, ultrasonographic cervical length measurement are the methods used in predicting preterm labor ${ }^{(4,5)}$. Currently, ultrasonographic cervical length measurement is the most frequently used method in clinical practice for the prediction of preterm labor ${ }^{(6,7)}$.

There is evidence to support the view that activation of the fetal hypothalamic-pituitary-adrenal axis, and the cross talk between a variety of placental and fetal adrenal gland endocrine signaling pathways play an important role in initiation of the normal parturition process ${ }^{(8)}$.

Biochemical activation causes increased dehydroepinadrosterone-sulfate production in the central zone of the fetal adrenal gland (fetal zone). Accordingly the whole fetal adrenal gland increases in size and this increase is predominantly due to significant enlargement of the central fetal zone ${ }^{(9)}$. Arguments in support of this process have been provided by an autopsy study, which demonstrated that neonates that delivered in the setting of idiopathic PTB had significantly higher adrenal gland weight than those that delivered secondary to fetal/maternal hemorrhage ${ }^{(\mathbf{1})}$.

The recent studies conducted in this context revealed that the ultrasound-measured fetal adrenal gland volume is highly predictive of preterm birth, independent of expected birth weight and gestational week ${ }^{(\mathbf{1}-10)}$. Hence, this study aimed at comparing the fetal adrenal gland FZD/TGD ratios in preterm and term labor through ultrasonographic assessment.

\section{PATIENTS AND METHODS}

The study was carried out on 66 patients attending Outpatients and Emergency Unit of Gynecology and Obstetrics Department, from December 2019 to December 2020. Thirty three preterm pregnant women at 29-36 weeks of gestation with single pregnancy were admitted to the hospital with the clinical diagnosis of preterm labor. On the other hand, a control group of 33 pregnant women between 37 and 40 weeks with term 
pregnancy were included in this study.

\section{Exclusion criteria:}

Placenta previa, diabetes mellitus, gestational diabetes, maternal heart disease, hypertensive disorders, abruption placenta, multiple pregnancies, polyhydramnios, oligohydramnios, existence of anemia, fetal distress, FGR, stillborn fetuses and fetal abnormality incompatible with life.

\section{Inclusion criteria:}

The existence of regular, effective $(45-50 \mathrm{mmHg})$ uterine contractions, 4 in 20 minutes or 6 in 60 minutes, cervical dilatation of $\geq 2 \mathrm{~cm}$ or the existence of a cervical change during observations and premature rupture of membranes that were detected through sterile speculum examination.

The entire pregnant women undergoing systemic and obstetric examination were initially assessed by routine transabdominal ultrasonography $(3.5 \mathrm{MHz}$ convex abdominal prob, LOGIQ V series S/W function, examination was done by the same examiner). During ultrasonography, fetal biometric measurements were performed, fetal weight has been calculated and the relevant gestational ages were verified.

Fetal adrenal gland was assessed. Early term pregnancy and ultrasonographies were used to detect the week of gestation of those who do not know their last menstrual periods. The age, existing internal diseases, gravida, parity, preterm labor history and history of rupture membrane were queried and recorded. The fetal adrenal gland was assessed by means of abdominal probe passing from the transverse plane, where fetal renal structures are observed, to the sagittal plane at a 90 degrees angle. Hyperechogenic central portion of the fetal adrenal gland in the sagittal plane, Fetal Zone Depth (FZD) in anterior-posterior plane, and Total Gland Depth (TGD) in the same plane were measured in millimeters.

The FZD/TGD ratio helped us to obtain the data underlying our study.

\section{Ethical approval:}

Before the study, a written consent form was received from the hospitals following the approval of the Ethics Committee of Faculty of Medicine Menoufia University.

\section{Data analysis}

Data were analyzed using IBM( $\odot$ SPSS $\odot$ Statistics version 23 (IBM@ Corp., Armonk, NY) and XLSTAT $\odot$ Version 2016.02.28451 (Addinsoft $\odot$, Paris, France). Continuous numerical variables were presented as mean and SD and inter-group differences are compared using the unpaired t test.

Categorical variables are presented as number and percentage and differences are compared using the Pearson chi-squared test or Fisher's exact test. Ordinal data are compared using the chi-squared test for trend. P value $\leq 0.05$ is considered significant.

\section{RESULTS}

Totally 66 pregnant women were included in the study. Two assessment groups 33 preterm and 33 term cases as a comparison group were created. Preterm birth taken place between $29-36^{\text {th }}$ weeks while term births taken place between $37-40^{\text {th }}$.

No difference was found between the two groups in respect to age and number of pregnancies $(\mathrm{P}>0.05)$. Yet, preterm birth history, rupture of membrane, cervical dilatation and mode of delivery were higher in preterm group $(\mathrm{P}<0.05)$ (Table 1$)$.

Table (1): Descriptive statistics for the study population

\begin{tabular}{|l|c|}
\hline Variable & Value \\
\hline Parity & $9(13.6 \%)$ \\
\hline P0 & $14(21.2 \%)$ \\
\hline P1 & $23(34.8 \%)$ \\
\hline P2 & $15(22.7 \%)$ \\
\hline P3 & $4(6.1 \%)$ \\
\hline P4 & $1(1.5 \%)$ \\
\hline P5 & $57(86.4 \%)$ \\
\hline History of PTL & $9(13.6 \%)$ \\
\hline Negative & $46(69.7 \%)$ \\
\hline Positive & $20(30.3 \%$ \\
\hline Membranes & $0.6 \pm 1.3(0.0$ to 5.0$)$ \\
\hline Intact & \\
\hline Ruptured & \\
\hline $\begin{array}{l}\text { Cervical dilatation } \\
\text { (cm) }\end{array}$ & $0.28 \pm 0.12(0.07$ to 0.57$)$ \\
\hline FZD (cm) & $0.72 \pm 0.21(0.30$ to 1.09$)$ \\
\hline TGD (cm) & $0.394 \pm 0.136(0.194$ to 0.635$)$ \\
\hline TZD/TGD ratio & \\
\hline Mode of delivery & $23(34.8 \%)$ \\
\hline Vaginal & $36.0 \pm 3.2(29.0$ to 40.0$)$ \\
\hline Caesarean & \\
\hline $\begin{array}{l}\text { Gestational age } \\
\text { (weeks) }\end{array}$ & $29(43.9 \%)$ \\
\hline Timing of delivery & \\
\hline Term & \\
\hline Preterm & \\
\hline
\end{tabular}

Of the women evaluated in this study, 27 patient delivered preterm (delivered within 7days (43.9\%), 7 cases had history of PTL and 17 cases had PROM (Figure 1). 


\section{Main Outcomes}

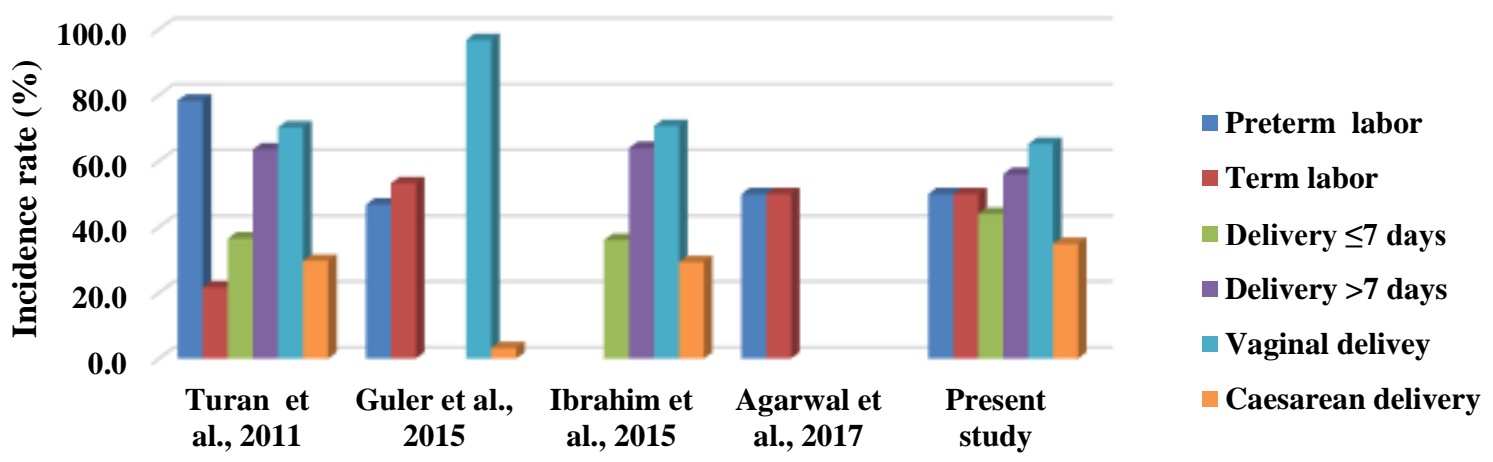

Outome

Figure (1): Incidence of main outcomes (preterm labor, term labor, delivery $\leq 7$ days, delivery $>7$ days, vaginal delivery, cesarean delivery) estimated by the current study as contrasted with those estimated from previous studies. Regarding mode of delivery, 14 cases were vaginal and 15 cases were caesarean (Table 2).

Table (2): Characteristics of patients who underwent preterm or term delivery

\begin{tabular}{|c|c|c|c|}
\hline Variable & Term delivery $(n=37)$ & Preterm delivery $(n=29)$ & P-value \\
\hline Parity & & & $0.773 \dagger$ \\
\hline$P 0$ & $6(16.2 \%)$ & $3(10.3 \%)$ & \\
\hline$P 1$ & $10(27.0 \%)$ & $4(13.8 \%)$ & \\
\hline$P 2$ & $7(18.9 \%)$ & $16(55.2 \%)$ & \\
\hline$P 3$ & $9(24.3 \%)$ & $6(20.7 \%)$ & \\
\hline$P 4$ & $4(10.8 \%)$ & $0(0.0 \%)$ & \\
\hline$P 5$ & $1(2.7 \%)$ & $0(0.0 \%)$ & \\
\hline History of PTL & & & $0.036 t$ \\
\hline Negative & $35(94.6 \%)$ & $22(75.9 \%)$ & \\
\hline Positive & $2(5.4 \%)$ & $7(24.1 \%)$ & \\
\hline Membranes & & & $<0.001 \S$ \\
\hline Intact & $34(91.9 \%)$ & $12(41.4 \%)$ & \\
\hline Ruptured & $3(8.1 \%)$ & $17(58.6 \%)$ & \\
\hline Mode of delivery & & & $0.011 \S$ \\
\hline Vaginal & $29(78.4 \%)$ & $14(48.3 \%)$ & \\
\hline Caesarean & $8(21.6 \%)$ & $15(51.7 \%)$ & \\
\hline
\end{tabular}

Fetal adrenal gland enlargement (FZD/TGD ratio) was statistically significantly higher in preterm group compared to the term group $(52 \% \pm 5 \%$ vs. $29 \% \pm 9 \%$; $\mathrm{P}<0.001)$ (Table 3$)$.

Table (3): Comparison of FZD, TGD and FZD/TGD ratio in patients undergoing preterm or term delivery

\begin{tabular}{|c|c|c|c|c|c|c|c|}
\hline \multirow{2}{*}{ Variable } & \multirow{2}{*}{ Value } & \multirow{2}{*}{$\begin{array}{c}\text { Term } \\
\text { delivery } \\
(\mathbf{n}=37)\end{array}$} & \multirow{2}{*}{$\begin{array}{c}\text { Preterm } \\
\text { delivery } \\
(\mathbf{n}=29)\end{array}$} & \multirow{2}{*}{$\begin{array}{c}\text { Mean } \\
\text { Difference }\end{array}$} & \multicolumn{2}{|c|}{$95 \% \mathrm{CI}$} & \multirow{2}{*}{$\begin{array}{c}\text { P- } \\
\text { value } \dagger\end{array}$} \\
\hline & & & & & Lower & Upper & \\
\hline $\begin{array}{l}\text { Cervical dilatation } \\
(\mathrm{cm})\end{array}$ & $\begin{array}{c}0.6 \pm 1.3 \\
(0.0 \text { to } 5.0)\end{array}$ & $0.00 \pm 0.00$ & $1.48 \pm 1.72$ & -1.48 & -2.05 & -0.92 & $<0.001$ \\
\hline FZD $(\mathbf{c m})$ & $\begin{array}{c}0.28 \pm 0.12(0.07 \\
\text { to } 0.57)\end{array}$ & $0.22 \pm 0.08$ & $0.36 \pm 0.12$ & -0.14 & -0.19 & -0.08 & $<0.001$ \\
\hline TGD (cm) & $\begin{array}{l}0.72 \pm 0.21(0.30 \\
\text { to } 1.09)\end{array}$ & $0.76 \pm 0.22$ & $0.68 \pm 0.20$ & 0.08 & -0.03 & 0.18 & 0.148 \\
\hline FZD/TGD ratio & $\begin{array}{c}0.394 \pm 0.136 \\
(0.194 \text { to } 0.635)\end{array}$ & $0.29 \pm 0.09$ & $0.52 \pm 0.05$ & -0.23 & -0.26 & -0.19 & $<0.001$ \\
\hline
\end{tabular}

There is a direct positive relationship between cervical dilatation and preterm labor. Also there is a direct positive relationship between increases FZD that lead to increase FZD/TGD Ratio in patients undergoing preterm delivery. On ROC analysis, the area under the curve (AUC) for FZD, TGD or TZD/TGD ratio was $0.821,0.603$ and 0.983 . The ROC curve shows that the FZD sensitivity was $79 \%$ and specificity was $86 \%$. TGD sensitivity was $69 \%$ and specificity $54 \%$ (Figure 2). 


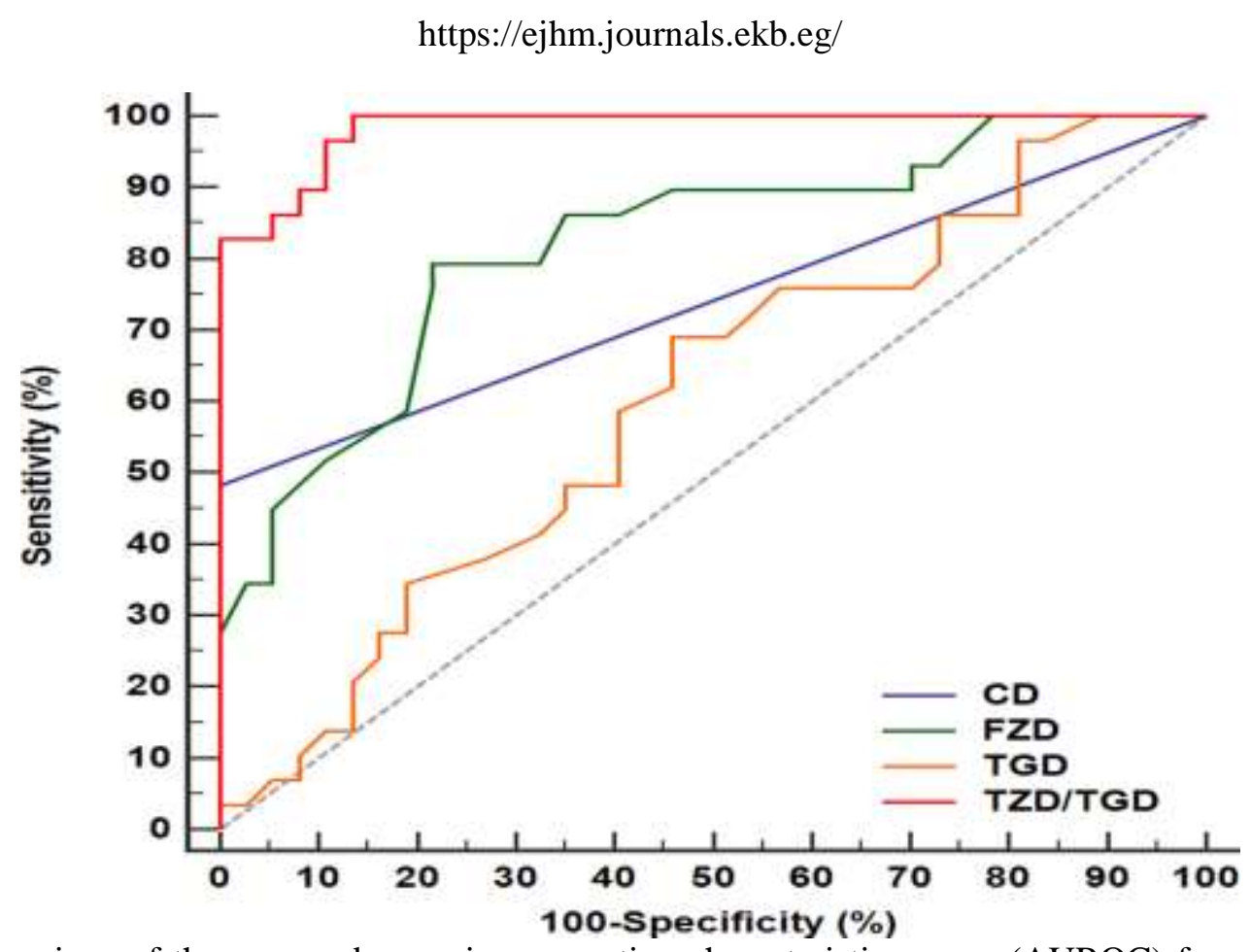

Figure (2): Comparison of the area under receiver-operating characteristic curves (AUROC) for cervical dilatation, FZD, TGD or TZD/TGD ratio.

The best cut off value of present study was FZD/TGD ratio with sensitivity $100 \%$ and specificity $86 \%$ in prediction of preterm birth as in other studies made (Figure 3).

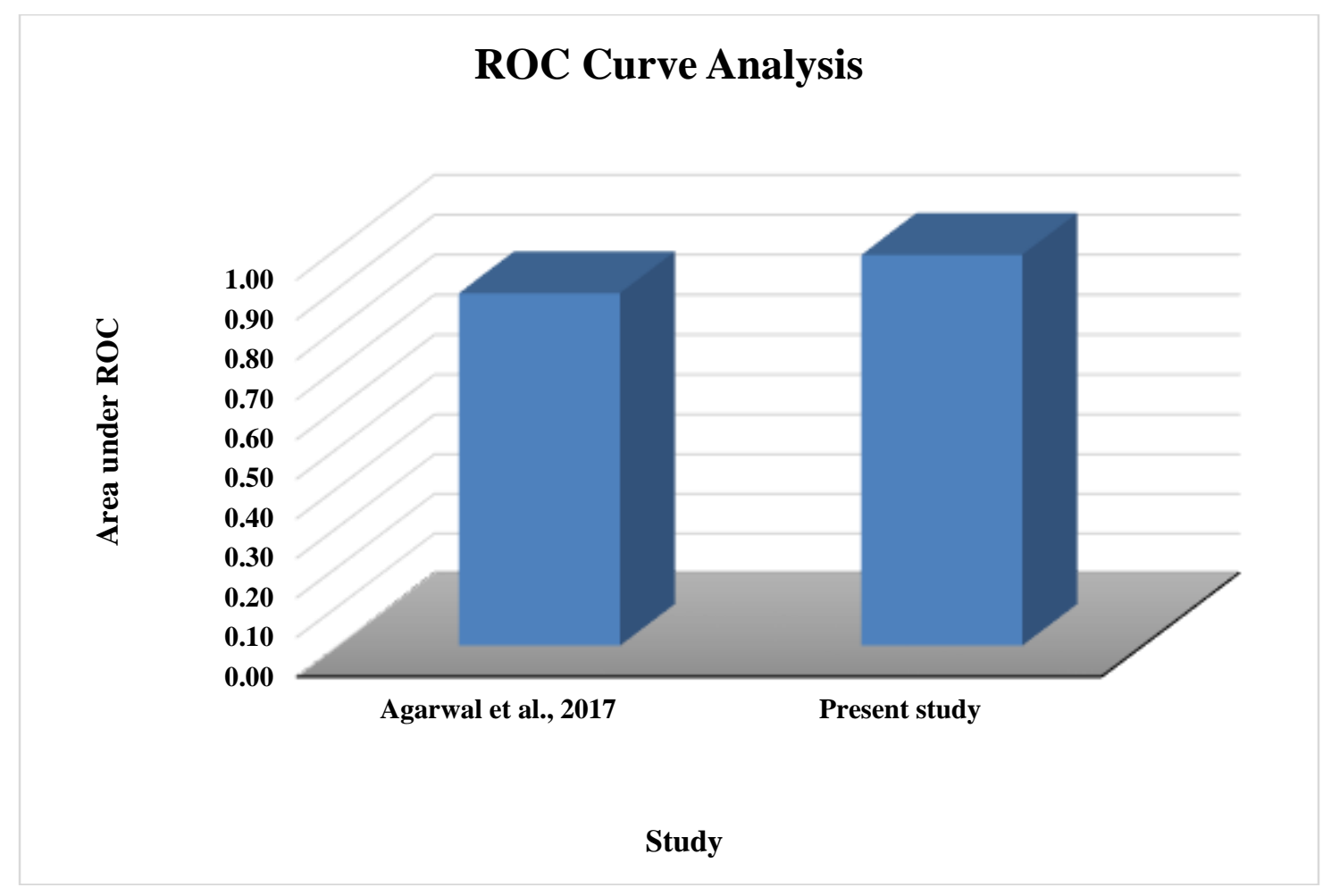

Figure (3): Area under ROC curve for prediction of preterm delivery using TZD/TGD Ratio estimated by the current study as contrasted with those estimated from previous studies

On the other hand PPV and NPP of FZD were $74 \%$ and $83 \%$ respectively. PPV and NPP of TGD were 54\% and $69 \%$ respectively (Table 4 ). 
Table (4): Receiver-operating characteristic (ROC) Curve for prediction of preterm delivery using cervical dilatation, FZD, TGD or TZD/TGD ratio

\begin{tabular}{|c|c|c|c|c|}
\hline \multirow[b]{2}{*}{ ROC index } & \multicolumn{4}{|c|}{ Predictor } \\
\hline & $\begin{array}{c}\text { Cervical } \\
\text { dilatation }\end{array}$ & FZD & TGD & TZD/TGD ratio \\
\hline AUC & 0.741 & 0.821 & 0.603 & 0.983 \\
\hline Standard error & 0.000 & 0.049 & 0.068 & 0.000 \\
\hline Lower bound $(95 \%)$ & 0.741 & 0.725 & 0.470 & 0.983 \\
\hline Upper bound (95\%) & 0.741 & 0.916 & 0.736 & 0.983 \\
\hline Cut-off & $2.0 \mathrm{~cm}$ & $0.290 \mathrm{~cm}$ & $0.740 \mathrm{~cm}$ & 0.402 \\
\hline Sensitivity & $48 \%$ & $79 \%$ & $69 \%$ & $100 \%$ \\
\hline Lower bound (95\%) & $31 \%$ & $61 \%$ & $51 \%$ & $86 \%$ \\
\hline Upper bound (95\%) & $66 \%$ & $90 \%$ & $83 \%$ & $100 \%$ \\
\hline Specificity & $100 \%$ & $78 \%$ & $54 \%$ & $86 \%$ \\
\hline Lower bound (95\%) & $89 \%$ & $62 \%$ & $38 \%$ & $71 \%$ \\
\hline Upper bound $(95 \%)$ & $100 \%$ & $89 \%$ & $69 \%$ & $94 \%$ \\
\hline PPV & $100 \%$ & $74 \%$ & $54 \%$ & $85 \%$ \\
\hline NPV & $71 \%$ & $83 \%$ & $69 \%$ & $100 \%$ \\
\hline $\mathrm{LR}+$ & $+\operatorname{Inf}$ & 3.67 & 1.50 & 7.40 \\
\hline LR- & 0.52 & 0.26 & 0.57 & 0.00 \\
\hline TP & 14 & 23 & 20 & 29 \\
\hline $\mathrm{TN}$ & 37 & 29 & 20 & 32 \\
\hline FP & 0 & 8 & 17 & 5 \\
\hline FN & 15 & 6 & 9 & 0 \\
\hline Sensitivity + Specificity & 1.483 & 1.577 & 1.230 & 1.865 \\
\hline Yonden $(\mathrm{J})$ index & 0.483 & 0.577 & 0.230 & 0.865 \\
\hline Accuracy & $77 \%$ & $79 \%$ & $61 \%$ & $92 \%$ \\
\hline
\end{tabular}

ROC $=$ receiver-operating characteristic, $\mathrm{AUC}=$ area under curve, $\mathrm{PPV}=$ positive predictive value, $\mathrm{NPV}=$ negative predictive value, $\mathrm{LR}+=$ likelihood ratio positive, $\mathrm{LR}-=$ likelihood ratio negative, $\mathrm{FN}=$ false negative, $\mathrm{FP}=$ false positive, $\mathrm{TN}=$ true negative, $\mathrm{TP}=$ true positive.

PPV and NPP of FZD/TGD ratio were $85 \%$ and $100 \%$ respectively that was the most accurate method in prediction of preterm labor as in other studies (Figure 4).

\section{Diagnostic Accuracy}

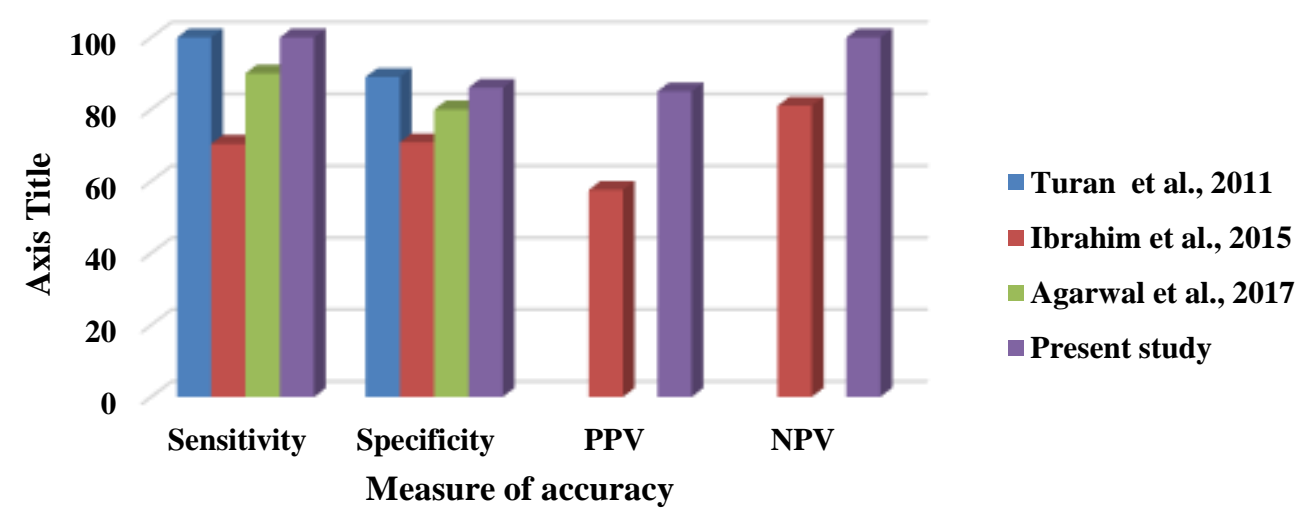

Figure (4): Diagnostic accuracy (sensitivity, specificity, PPV, NPV) for TZD/TGD ratio for prediction of preterm delivery estimated by the current study as contrasted with those estimated from previous studies.

On ROC analysis, the area under the curve (AUC) for prediction of preterm birth using cervical dilatation, FZD, TGD or FZD/TGD ratio. The best cut off value using FZD/TGD ratio (AUC=0,983) (Table 5). 
Table (5): Comparison of receiver-operating characteristic (ROC) Curves for prediction of preterm delivery using cervical dilatation, FZD, TGD or TZD/TGD ratio

\begin{tabular}{|c|c|c|c|c|c|}
\hline Variable & AUC & SE & $95 \% \mathrm{CI}$ & & \\
\hline $\mathrm{CD}$ & 0.741 & 0.047 & 0.619 to 0.841 & & \\
\hline FZD & 0.821 & 0.053 & 0.707 to 0.904 & & \\
\hline TGD & 0.603 & 0.070 & 0.475 to 0.721 & & \\
\hline TZD/TGD Ratio & 0.983 & 0.011 & 0.916 to 0.999 & & \\
\hline Comparison & $\triangle \mathrm{AUC}$ & $\mathrm{SE}$ & $95 \% \mathrm{CI}$ & z-statistic & P-value \\
\hline CD vs. FZD & 0.079 & 0.077 & -0.071 to 0.229 & 1.035 & 0.301 \\
\hline CD vs. TGD & 0.138 & 0.086 & -0.029 to 0.306 & 1.618 & 0.106 \\
\hline CD vs. TZD/TGD & 0.242 & 0.051 & 0.143 to 0.341 & 4.787 & $<0.0001$ \\
\hline FZD vs. TGD & 0.218 & 0.111 & -0.0003 to 0.435 & 1.958 & 0.0503 \\
\hline FZD vs. TZD/TGD & 0.163 & 0.049 & 0.066 to 0.260 & 3.290 & 0.001 \\
\hline TGD vs. TZD/TGD & 0.380 & 0.071 & 0.240 to 0.520 & 5.327 & $<0.0001$ \\
\hline
\end{tabular}

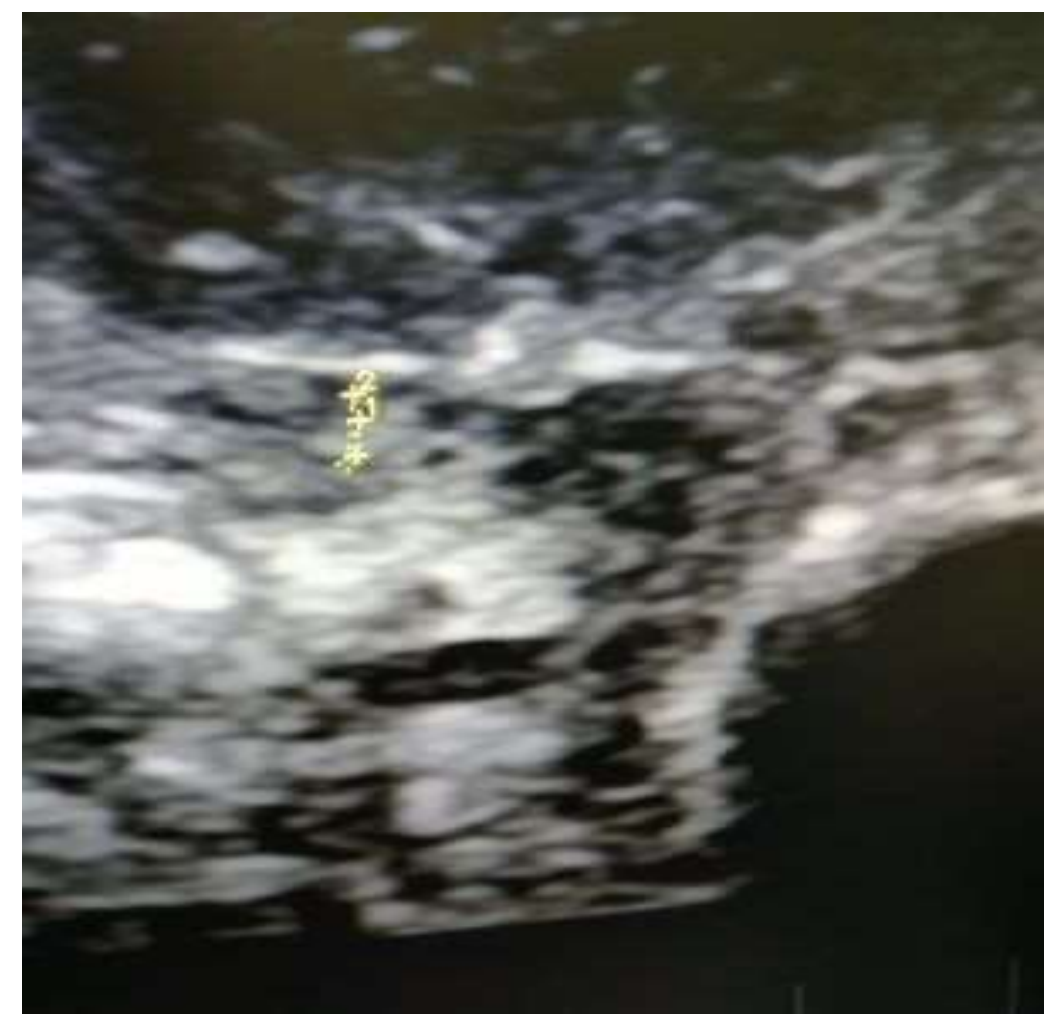

Figure (5): Female patients 29 years old p3 NVD 33 weeks admitted at emergency room, by premature rupture of membrane history of preterm labor in the last delivery by measuring fetal zone enlargement $=0.59$ and patient delivered $<7$ days from admission.

\section{DISCUSSION}

Preterm birth (PTB) remains a major cause of perinatal morbidity and mortality worldwide. Compared with term deliveries, early PTB $(<34$ weeks gestation) carries a 7-fold increased risk of neonatal death. Following PTB, survivors can experience significant long-term cognitive, behavioral, emotional, sensory, and motor deficits. ${ }^{(7)}$.

The studies conducted for long years focused on the assessment of maternal responses of the maternal mechanisms responsible for the etiology of premature labor. However, in clinical cases like preterm labor with raised fetal stress, the assessment of clinical results of hypothalamic-pituitary-adrenal axis, hormonal structures perpetuating this axis and this hormone stimulation recently become important. The focal point of this study, FZ of the fetal adrenal gland produces the precursors of the steroid hormones transforming into estrogen and other cortisol derivatives in the placenta. Fetal cortisol increases placental corticotropin-releasing hormone (CRH) and causes the release of PGE2 and PGF $2 \alpha$ of the placental endogenous birth labor different than the pituitary corticotropin releasing hormone. Considering the site of cortisol production reaching maximum levels in circulating maternal blood at 3436th weeks of pregnancy under the rules of adaptation to fetal stress with estrogen; one will see that the increase in FZ width of the preterm cases is an expected 
result $^{(3,11)}$.

In this study, we compared 29 preterm labor cases with 37 cases of spontaneous delivery at term. Though they seem to be the same in respect of mechanism and fetal response, preterm and term labors are actually far from being similar particularly when we consider the anatomical responses to hormonal effects like increment in depth of fetal adrenal gland. FZD/TGD ratio was higher in preterm birth group, compared to the term birth group $(\mathrm{P}<0.001)$. Examining the entire risk factors of preterm labor, all the reasons except for maternal age and ethnic origin contribute to the environment of a difficult intrauterine fetal growth and development. While this environment implies a mechanism of severe stress adaptation, the lack of such features in a term fetus accounts for the difference between the two comparisons.

The results obtained in our study propound that the ultrasonographic assessment of fetal adrenal gland and fetal zone enlargement may be beneficial for the cases presented with preterm labor symptoms. We think that these assessment results may provide rapid administration and treatment facilities with real indications for the patient groups admitted with preterm labor symptoms. For instance, one may encounter the fact of the implementation of unnecessary treatments as cerclage, tocolysis and steroids in cases of increased fetal zone enlargement.

The fetal adrenal gland volume in the risky group as an unusual method for preterm labor; fetal adrenal gland volumes of the deliveries before $37^{\text {th }}$ week, as an indicator of hypothalamic-pituitary-adrenal functioning were assessed by three-dimensional ultrasonography. Fifty three patients with preterm labor symptoms and 73 cases without symptoms were included in this study. Corrected adrenal gland volume for estimated fetal weight was measured and 422 $\mathrm{mm}^{3} / \mathrm{kg}$ was set as limit value. The probability of delivery within 5 days of the cases exceeding this value was statistically significant ${ }^{(1)}$. In another study of the same group, corrected adrenal gland volume for estimated feta weight, fetal zone enlargement of adrenal gland and cervical length were assessed by two and three dimensional ultrasonography. The ratio between whole gland depth and central fetal zone depth, vaginal ultrasound assessment of cervical length data and specificity and sensitivity of prediction of preterm labor of corrected fetal adrenal gland volume measurements within 7 days, were elaborated here, as in our study. As a result of 7-day follow-up of fetal zone enlargement independent from obstetrical history and tocolytic treatment, specificity and sensitivity levels were higher compared to the other two methods in respect of ending in preterm labor ${ }^{(1)}$

Agarwal et al. ${ }^{(12)}$ highlighted that fetal adrenal gland biometry and elastography of the antenatal cervix are beneficial in prediction of preterm birth. The correlation between SWS of the cervix, trans-abdominal
(TA) cervical length, and fetal adrenal enlargement is also demonstrated with the relative difference in their levels of accuracy. Also, they showed that FZE of both adrenal glands are in agreement with each other. Hence, this eliminated the ambiguity in adrenal gland selection and found that $\mathrm{cAGV}$ was higher in preterm deliveries with $67 \%$ sensitivity and $76 \%$ specificity at cut-off value of $415 \mathrm{~mm}^{3} / \mathrm{kg}$ ( $\left.\mathrm{p}<0.001\right)$, while enlargement of the fetal zone of the adrenal gland showed $90 \%$ sensitivity and $80 \%$ specificity at cut off value of $47.7 \%$, shear wave speed was $96.7 \%$ sensitivity and $87 \%$ specificity at cut off value of $2.87(\mathrm{~m} / \mathrm{s})$ and cervical length was $63 \%$ sensitivity and $47 \%$ specificity at cut off value of $2.0(\mathrm{~cm})$.

Ibrahim et al. (13) compare the diagnostic accuracies of 3D ultrasound measurements of $\mathrm{cAGV}$ and FZE as predictors of PTB with those of the currently recommended PTB prediction tests, such as ultrasound measurements of the $\mathrm{CL}$ and cervicovaginal fetal fibronectin (CVFF). Moreover, this was the first study to compare the above parameters in women who presented only with threatened PTL after excluding women with PROM to restrict the final statistical analysis entirely to the process of PTB. The sensitivity and specificity of CL as a predictor of PTB within 7 days of enrollment were $81.5 \%$ and $56.2 \%$ respectively, along with 51.2 \% PPV and 84.4\% NPV. The role of the short cervix in PTB pathogenesis is controversial. The sensitivity and specificity of CVFF as a predictor of PTB within 7 days of enrollment in this study were 74.1 and $68.8 \%$ respectively, with 57.1\% PPV and 82.5 $\%$ NPV. While, a cAGV of $\geq 405 \mathrm{~mm}^{3}$ was associated with the highest sensitivity $(92.6 \%)$ and specificity (95.8\%), as well as higher PPV (92.6\%) and NPV (95.8 $\%$ ), compared to those of FZE (92.6 and $89.6 \%$ respectively) and CL (81.5 and $56.2 \%$ respectively).

Guler et al. (2) compared the diagnostic accuracies of ultrasound measurements of CL and FZE as predictors of PTB. 29 preterm labor cases compared to 33 cases of spontaneous delivery at term. FZD/TGD ratio was higher in preterm birth group compared to the term birth group $(\mathrm{P}<0.001)$, while $\mathrm{P}$ value of cervical length was 0.27 . This study propound that the ultrasonographic assessment of fetal adrenal gland and fetal zone enlargement may be beneficial for the cases presented with preterm labor symptoms.

The results obtained in our study propound that the ultrasonographic assessment of fetal adrenal gland and fetal zone enlargement may be beneficial for the cases presented with preterm labor symptoms. We think that these assessment results may provide rapid administration and treatment facilities with real indications for the patient groups admitted with preterm labor symptoms. For instance, one may encounter the fact of the implementation of unnecessary treatments as cerclage, tocolysis and steroids in cases of increased fetal zone enlargement (Figure 5). 


\section{CONCLUSION}

2D ultrasound evaluation of the fetal zone is a noninvasive clinical tool, which holds promise to change the clinical practice and management of PTB. Two-dimensional measurement of the depth of the adrenal fetal zone offers the potential to accurately predict PTB within 7 days.

\author{
Abbreviations: \\ AUC: area under the curve \\ cAGV: corrected adrenal gland volume \\ FGR: fetal growth retardation \\ FZ: fetal zone \\ FZD: fetal zone depth \\ FZD: fetal zone enlargement \\ NPP: negative predictive value \\ PPV: positive predictive value \\ PTB: preterm birth \\ PROM: premature rupture of membrane \\ TGD: total gland depth
}

\section{REFERENCES}

1. Turan O, Turan S, Funai E et al. (2011): Ultrasound measurement of fetal adrenal gland enlargement: an accurate predictor of preterm birth. American Journal of Obstetrics Gynecology, 204 (4): 311-15.

2. Guler A, Pehlivan H, Cakmak B et al. (2015): Assessment of fetal adrenal gland enlargement in term and preterm labor cases. International Journal of Research in Medical Sciences, 3 (5): 1035-1040.

3. McIntosh J, Helen F, Berghella V et al. (2016): The role of routine cervical length screening in selected high- and low-risk women for preterm birth prevention. Am J Obstet. Gynecol., 215 (3): 2-7.
4. Song J, Lee K, Kim M et al. (2012): Cervical funneling after cerclage in cervical incompetence as a predictor of pregnancy outcome. The Journal of Maternal-Fetal \& Neonatal Medicine, 25 (2): 147-150.

5. Amanda R, Anju S, Vincenzo B et al. (2016): Overview of Cervical Insufficiency: Diagnosis, Etiologies, and Risk Factors. Clinical Obstet. Gynecol., 59 (2): 237-240.

6. Ville Y, Rozenberg $\mathbf{P}$ (2018): Predictors of preterm birth. Best Practice \& Research Clinical Obstetrics \& Gynaecology, 52: 23-32.

7. Sharvit M, Weiss R, Paz Y et al. (2017): Vaginal examination vs. cervical length which is superior in predicting preterm birth. Journal of Prenatal Medicine, 45 (8): 977-983.

8. Navathe R, Berghella V (2016): Progesterone as a tocolytic agent for preterm labor: a systematic review. Current Opinion in Obstetrics and Gynecology, 28 (6): 464-469.

9. Yang X, Zhen Y, Liu L (2020): The Development and Functional Regulation of the Fetal Adrenal Cortex. Yangtze Medicine, 4 (4): 254-276.

10. Heese S, Hammer K, Möllers M et al. (2018): Adrenal gland size in growth restricted fetuses. Journal of Perinatal Medicine, 46(8): 900-904.

11. Areia A, Moura P (2015): Amniotic Membrane in Health and Disease: An Obstetrical Perspective. Amniotic Membrane: Springer, Pp: 77-101.

12. Agarwal S, Agarwal A, Joon $P$ et al. (2018): Fetal adrenal gland biometry and cervical elastography as predictors of Preterm birth: A comparative study. Ultrasound, 26 (1): 54-62

13. Ibrahim M, Sherif A, El-Kady M et al. (2015): Can three-dimensional ultrasound measurement of fetal adrenal gland enlargement predict preterm birth? Arch Gynecol Obstet., 292 (3): 569-78. 